northern Europe, especially the North Sea where it is frequently found stranded on the shore in winter.

Brama is the most numerous of the bramid genera and seven species are recognized. In the Atlantic, Mead finds that $B$. brama is confined to the northern temperate Atlantic and Mediterranean; another species, $B$. dussumieri, replaces it to the south (and also occurs in the Indian and Pacific Oceans). A third species is found in the Caribbean Sea and the tropical western Atlantic. The two species $B$. brama and $B$. dussumieri seem to have mutually exclusive ranges based, Mead deduces, on the limits set by temperature, that of $23.8^{\circ} \mathrm{C}$ being critical.

Clearly much remains to be learned of bramid biology. The north Atlantic Brama brama is relatively well known, but its season and area of breeding are still unknown and its diet is unstudied; all of the other bramids are less well known than this. Mead's study, with its analysis of the literature (painstakingly detailed through to the mid-nineteensixties, but strangely only a few general works cited later) and of a large number of specimens, provides a very useful guide for further work.

\section{DNA STRUCTURE}

\section{Geometrical Versatility}

from our Molecular Biology Correspondent

THE crystallographer's hobgoblin, that the structure in his crystal or fibre is not exactly that of the same molecule in solution, has been of late manifesting itself rather strongly in regard to DNA. Until recently three structures were recognized in fibres, and these-the $A$, $B$ and $C$ forms-could be made to interconvert in response to changes in humidity and salt. The evidence is that in dilute solution DNA is in the B form, which is found in fibres at high humidity. Just how this relates to DNA in the cell, be it in the nucleohistone of mammalian chromosomes, in nucleoprotamine or in phage heads, is by no means clear, and there are some indications that the answer may not be altogether simple.

The optical activity of two-stranded DNA is highly sensitive to the helix geometry, and therefore serves as a delicate, if only in a rudimentary sense interpretable, marker for its response to changes in its environment. It has been abundantly demonstrated that in dispersed nucleohistone the circular dichroism differs from that of DNA in free solution, and resembles rather closely that of the $\mathrm{C}$ form. In complexes of DNA with some histone fractions, on the other hand, startingly different curves are obtained, with inversion of Cotton effects. Disregarding the awkward possibility of contributions from optical artefacts, which are difficult to rule out in work on particulate samples, it seems as though the phenomena in themselves may be highly specific.

A recent example concerns the complex of DNA with the lysine-rich histone, FI. Gottesfeld et al. (Biochemistry, 11, 1422; 1972) find that the inversion of the DNA circular dichroism, which occurs in this system, is provoked more readily by the homologous F1 fraction than that from other animal sources, but that this species specificity is lost after purification of the histones by ion-exchange chromatography. Crudely interpreted, this means that the specificity of interaction is promoted by another kind of molecule. Again, the smaller circular dichroism disturbance induced in DNA by histone IV is modified by calcium ions (Wagner and Vandergrift, ibid., 1431).

It is not, however, only in these complex systems that the optical properties of DNA undergo large changes. High salt concentrations and the addition of non-aqueous solvents can encompass such effects in dilute isotropic solutions, and it has long been known that increasing temperature, below the melting region, causes physical changes in DNA, which are manifested in various ways, including an increase in circular dichroism, and as Palecek and Fric (Biochem. Biophys, Res. Commun., 47,

\section{GEOLOGY \\ Ulster Surveyed}

THE series of handbooks on the "Regional Geology of Great Britain" has for some 30 years been a much valued source of reference for both amateur and professional geologists. The Geological Survey has now extended the series to Northern Ireland (HMSO, 50p) and the volume effectively shows the variety of geological features of all types in the region-from the Moinian to the Quaternary (with the exception of the Cambrian) together with many examples of glacial and recent phenomena. It is expected that a new map of Ulster on the scale of $1 / 250,000$ will be published soon after this handbook.

1262 ; 1971) report, a progressive increase in polarographic reducibility. Gennis and Cantor (J. Mol. Biol., 65, 381 ; 1972) found that the circular dichroism change is largest in poly dAT, that it occurs in glucosylated DNA, and that it is independent of salt concentration. It is thus evidently not to be identified with a transition to the $\mathrm{A}$ form, but represents some kind of geometrical adjustment.

An interesting investigation of the

\title{
Transcription and Excision of Prophage Lambda
}

IN next Wednesday's Nature New Biology (July 12) Davies, Dove, Inokuchi, Lehman and Roehrdanz describe a set of ingenious experiments with lambda lysogens which clearly indicate that the rate of excision of lambda prophages from the chromosomes of their Escherichia coli hosts is regulated at least in part by local transcription of the prophage DNA. This finding, together with the previous observation, made by this and other groups, that the initiation of DNA replication is intimately dependent upon transcription of certain regions of the DNA to be replicated, indicates that the control of bacteriophage, and probably bacterial gene replication, gene excision and gene expression, are integrated processes considerably more subtle than most people have envisaged.

Davies et al. exploited to the full the sophisticated techniques of genetic manipulation which are now available to the bacterial geneticists, especially to those who work with phage lambda. They constructed a strain of $E$. coli carrying a lambda prophage which because of mutation cannot make the so-called $\mathbf{N}$ gene product which is required for the transcription of most of the lambda genome. They then super- infected these lysogens with one or other of two helper phages and measured indirectly the excision of the defective lambda prophage genome.

Both helper phages provide all the enzymes required for the excision of the prophages and their subsequent replication as independent genomes, but only one of the two sorts of superinfecting helper phages used provides $\mathbf{N}$ gene product and therefore allows transcription of the lambda prophage before excision.

Results of this experiment, together with a variety of controls which rule out alternative explanations, lead to the conclusion that only when the prophage genome is being transcribed can it be excised to any significant extent. In short, local transcription directly changes the rate of excision, perhaps by altering the conformation or intracellular location of the prophage genome.

Any model to account for the control of excision must, therefore, subsume the mechanisms of the regulation of transcription, and although it may be convenient for experimentalists to regard DNA replication, transcription and translation as separate processes they are probably all closely interwoven. 